\title{
A polynomial algorithm for the Hamiltonian cycle problem in semicomplete multipartite digraphs
}

\author{
Jørgen Bang-Jensen* $\quad$ Gregory Gutin ${ }^{\dagger} \quad$ Anders Yeo ${ }^{\ddagger}$
}

\begin{abstract}
We describe a polynomial algorithm for the Hamiltonian cycle problem for semicomplete multipartite digraphs. The existence of such an algorithm was conjectured in [16] (see also [15]).
\end{abstract}

\section{Introduction}

A semicomplete multipartite digraph is a digraph $D=(V(D), A(D))$ for which the vertices of $V(D)$ can be partitioned into a number $k \geq 2$ of subsets (called colour classes) such that every pair of vertices from the same colour class are non-adjacent and every pair of vertices from different colour classes are adjacent (i.e. there is at least one arc between them). In Figure 1, a semicomplete 3-partite digraph $H$ is depicted. The colour classes are $\left\{x_{1}, x_{2}, x_{3}\right\},\left\{y_{1}, y_{2}, y_{3}\right\},\{z\}$. The following is a Hamiltonian cycle in $H: z x_{3} y_{3} x_{1} y_{1} x_{2} y_{2} z$.

Two well-known special cases are semicomplete digraphs (when $k=|V(D)|$ ) and semicomplete bipartite digraphs (when $k=2$ ). It is well-known that every strongly connected semicomplete digraph is Hamiltonian [9] and that a Hamiltonian cycle in a strong semicomplete digraph on $n$ vertices can be found in time $O\left(n^{2}\right)$ [18]. For semicomplete bipartite digraphs strong connectivity by itself is not enough to guarantee the existence of a Hamiltonian cycle. Here we need to require another, obviously necessary, condition that $D$ has a factor, i.e., a spanning collection of vertex-disjoint cycles. It was shown in [13] (see also [17]) that a semicomplete bipartite digraph is Hamiltonian if and only if it is strong and has a factor. It follows from the proof in [13] (see also [19]) that the existence of a Hamiltonian cycle in a semicomplete bipartite digraph on $n$ vertices can be checked in time $O\left(n^{2}+M(n)\right)$ where $M(n)$ is the time required to decide the existence of a perfect matching in a bipartite graph and find one if it exists.

*Department of Mathematics and Computer Science, Odense University, DK-5230, Odense, Denmark

${ }^{\dagger}$ Department of Mathematics and Statistics, Brunel University, Uxbridge, Middlesex UP8 3PH United Kingdom

${ }^{\ddagger}$ Department of Mathematics and Comptuter Science, Odense University, DK-5230, Odense, Denmark 


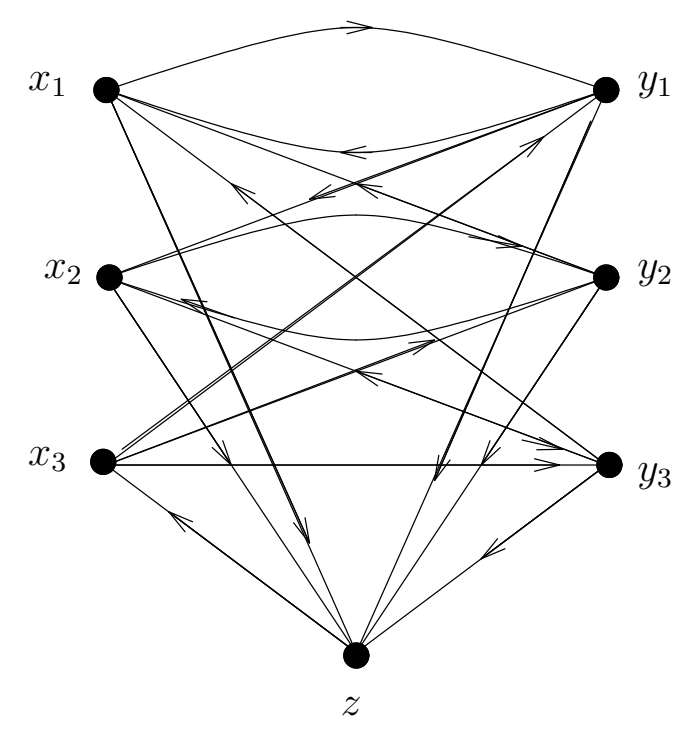

Figure 1: A semicomplete 3-partite digraph $H$.

Another special class of semicomplete multipartite digraphs for which a characterization of Hamiltonicity as well as a polynomial algorithm to find such a cycle (if it exists) was known, is the class of extended semicomplete digraphs (also called ordinary semicomplete multipartite digraphs in [14]). This is the class of digraphs one can obtain by starting from some semicomplete digraph by replacing each vertex $x$ by an independent set of vertices $I_{x}$ and adding all possible arcs from one such set $I_{x}$ to another $I_{y}$ if and only if the original digraph contained the arc $x \rightarrow y$. For extended semicomplete digraphs the same characterization as for semicomplete bipartite digraphs holds and a Hamiltonian cycle can be found within the same time bound as for semicomplete bipartite digraphs [14].

The complexity of the Hamiltonian cycle problem for general semicomplete multipartite digraphs has attracted the interest of several researchers and has been open for quite some time (it was first brought to the attention of the first author in a private communication with $\mathrm{C}$. Thomassen in 1987). While this problem has resisted a number of attempts to solve it (see e.g. [3, 22]), the Hamiltonian path problem has a surprisingly elegant solution for semicomplete multipartite digraphs. It was shown in [12] that a semicomplete multipartite digraph $D$ has a Hamiltonian path if and only if it has a path $P$ such that $D-V(P)$ has a factor and a Hamiltonian path can be found if one exists in time $O\left(n^{2}+M(n)\right)$ where $n$ is the number of vertices and $M(n)$ is as defined above (we remark that the proof of this result is non-trivial). Another characterization which is valid only for semicomplete multipartite digraphs with at most two vertices in each colour class can be found in [10]. In contrast to the nice characterization of Hamiltonian paths in semicomplete multipartite digraphs, it was shown in [3] that there is no $k$ such that every $k$-strong semicomplete multipartite digraph with a factor is Hamiltonian (a digraph $D$ is $k$-strong if $D$ has at least $k+1$ vertices and $D-X$ is strongly connected for every subset $X \subset V(D)$, where $|X| \leq k-1)$.

It turns out that the key to solving the Hamiltonian cycle problem for semicomplete 
multipartite digraphs lies in understanding the structure of reducible factors. A factor $C_{1} \cup C_{2} \cup \ldots \cup C_{k}$ of a digraph $D$ is reducible if $D$ has some other factor $C_{1}^{\prime} \cup C_{2}^{\prime} \cup \ldots \cup C_{r}^{\prime}$, $r<k$ such that for every $C_{i}$, there is some $C_{j}^{\prime}$ with $V\left(C_{i}\right) \subseteq V\left(C_{j}^{\prime}\right)$. (In Figure 1 the digraph $H$ contains a factor $\mathcal{F}=C_{1} \cup C_{2} \cup C_{3}$, where $C_{i}=x_{i} y_{i} x_{i}, i=1,2, C_{3}=x_{3} y_{3} z x_{3}$ and the factor $\mathcal{F}^{\prime}=C_{1}^{\prime} \cup C_{2}^{\prime}$ where $C_{1}^{\prime}=x_{1} y_{1} x_{2} y_{2} x_{1}$ and $C_{2}^{\prime}=x_{3} y_{3} z x_{3}$. The factor $\mathcal{F}$ can be reduced to the factor $\mathcal{F}^{\prime}$ which in turn can be reduced to a Hamiltonian cycle of $H$ ). In [3] the first such step was taken and a sufficient condition for Hamiltonicity of semicomplete multipartite digraphs, formulated in terms of reducible factors, was obtained (see Theorem 3.4). Recently the third author was able to refine the condition of Theorem 3.4 in such a way that we now have a very detailed understanding of reducible factors in semicomplete multipartite digraphs [21] (see Theorem 3.7).

Theorem 3.7 has many important consequences [21]:

- Every regular semicomplete multipartite digraph is Hamiltonian.

- If the size of the largest independent set in a $k$-strong semicomplete multipartite digraph $D$ is at most $2 k$ and $D$ has a factor, then $D$ is Hamiltonian.

- Any set of $k$ vertices in a $k$-strong semicomplete multipartite digraph lie on a common cycle.

For yet another application of Theorem 3.7 see [4].

The purpose of this paper is to give a constructive proof for the existence of a polynomial algorithm for the Hamiltonian cycle problem for semicomplete multipartite digraphs (Theorem 5.3). The algorithm is highly non-trivial and relies heavily on Theorem 3.7, the main result of [21]. We have not tried to optimize the complexity of the algorithm since we found it was quite complicated to describe and prove the correctness of the algorithm, even without this goal in mind. For technical reasons we prove the existence of a polynomial algorithm for a slightly more general problem (see note 4.3).

\section{Terminology}

We shall assume that the reader is familiar with the standard terminology on digraphs and refer to [8] for terminology not discussed here. Every cycle and path is assumed simple and directed.

In this section, $D$ denotes a digraph (in the whole paper we use $n$ to denote the number of vertices in the digraph currently studied), $x$ and $y$ are distinct vertices of $D, X$ and $Y$ are disjoint sets of vertices in $D$. D is Hamiltonian if it contains a Hamiltonian cycle, namely a cycle with $n$ vertices. A $k$-path-cycle subgraph $\mathcal{F}$ of $D$ is a collection of $k$ paths $P_{1}, P_{2}, \ldots, P_{k}$ and a number, $s \geq 0$, of cycles $Z_{1}, Z_{2}, \ldots, Z_{s}$ in $D$ such that no two of $P_{1}, P_{2}, \ldots, P_{k}, Z_{1}, Z_{2}, \ldots, Z_{s}$ have a vertex in common. We shall write $\mathcal{F}=P_{1} \cup P_{2} \cup \ldots \cup P_{k} \cup Z_{1} \cup Z_{2} \cup \ldots \cup Z_{s}$ (we always list the paths before the cycles.) If $k=0$ then $\mathcal{F}$ is a cycle subgraph. A factor is a spanning cycle subgraph, i.e one that covers all vertices of $D$.

If there is an arc from $x$ to $y$ then we say that $x$ dominates $y$ and write $x \rightarrow y$. We write $X \rightarrow Y$ if $x \rightarrow y$ for every pair $x \in X, y \in Y$ of adjacent vertices. If $X \rightarrow Y$, then 


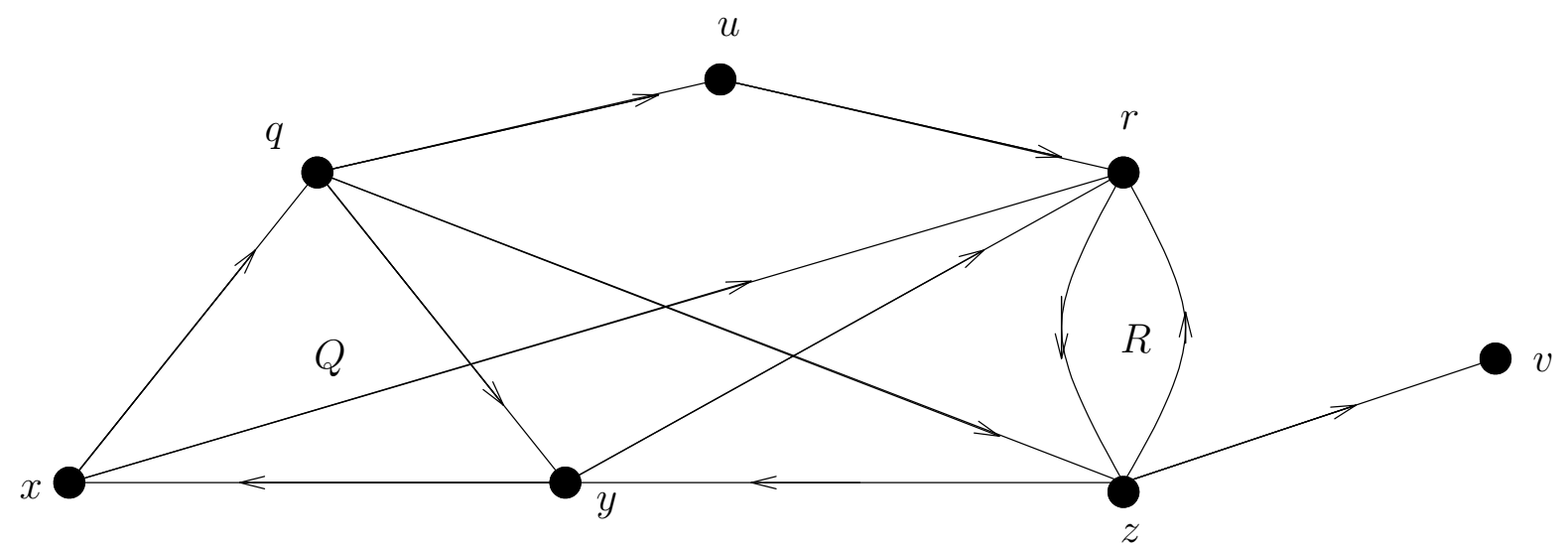

Figure 2: A digraph $D$ with two subgraphs $Q, R$ such that $Q \simeq>R$. Here $Q=\{q, x, y\}$ and $R=\{r, z\}$.

there may be a pair $x \in X, y \in Y$ such that both $x \rightarrow y$ and $y \rightarrow x$. Sometimes, we wish to exclude that possibility: We write $X \Rightarrow Y$ ( $X$ strongly dominates $Y$ ) if $X \rightarrow Y$ and there is no arc from $Y$ to $X$.

Let $R$ and $Q$ be disjoint subdigraphs of $D$. We write $Q \simeq>R$ if

1) $Q$ and $R$ contain vertices $q$ and $r$, respectively, such that $q \Rightarrow V(R)$ and $V(Q) \Rightarrow r$;

2 ) every vertex in $Q$ dominates a vertex in $R$ and every vertex of $R$ is dominated by a vertex of $Q$ (see Figure 2 for an example).

A path starting at $x$ and terminating at $y$ is an $(x, y)$-path. An $(x, y)$-path $P$ is a $(Q, R)$-path if $x \in V(Q), y \in V(R)$ and $|V(P) \cap(V(Q) \cup V(R))|=2$. Let $\mathcal{F}$

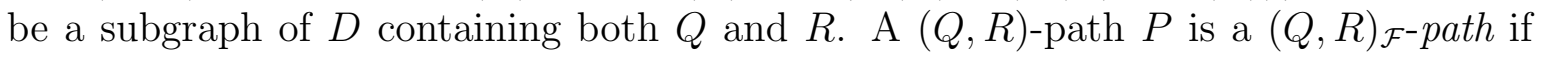
$|V(P) \cap V(\mathcal{F})|=2$, i.e. only the first and last vertex of $P$ is in $V(\mathcal{F})$. For example, in Figure 2 the path qur is a $(Q, R)_{\mathcal{F}}$-path, where $\mathcal{F}$ is the subdigraph induced by $V(Q) \cup V(R)$.

$D$ is strongly connected (or just strong) if there exists an $(x, y)$-path in $D$ for every ordered pair of distinct vertices $\{x, y\}$ of $D$. We say that $D$ is $k$-strong if $|V(D)| \geq k+1$ and $D-X$ is strongly connected for all $X \subset V(D)$ with $|X| \leq k-1$.

If $P$ is a path containing a subpath from $x$ to $y$ we let $P[x, y]$ denote that subpath. Similarly, if $C$ is a cycle containing vertices $x$ and $y, C[x, y]$ denotes the subpath of $C$ from $x$ to $y$. $D\langle Q\rangle$ is the subgraph induced by the vertices in $Q$. Let $C$ be a cycle containing $z$ and a subset $X \subseteq V(D)$. Then, $z_{C}^{+}\left(z_{C}^{-}\right)$denotes the successor (predecessor, resp.) of $z$ on $C$. Moreover, $X_{C}^{+}=\left\{x_{C}^{+}: x \in X\right\}, X_{C}^{-}=\left\{x_{C}^{-}: x \in X\right\}$. When the cycle $C$ is determined from the context, we shall usually omit the subscript $C$.

If $Y$ is a subset of the vertices of a digraph $D$ and $H$ is a subdigraph of $D$ such that $Y \subset V(H)$, then $H$ covers $Y$. 


\section{Preliminary results and outline of the main ideas}

We start with a few results on efficient construction of cycle subgraphs covering specified vertices.

Lemma 3.1 Given any digraph $D=(V(D), A(D))$ on $n$ vertices and $m$ arcs and a subset $W \subseteq V(D)$, in time $O(n m)$, we can either find a cycle subgraph $\mathcal{F}$ which covers $W$ or show that such a subgraph does not exist.

Proof: Let $D=(V(D), A(D))$ and $W$ be given. Form a network $N$ with lower bounds and capacities on the arcs in the following way: $V(N)=\left\{x^{\prime}, x^{\prime \prime} \mid x \in V(D)\right\}$. $A(N)=\left\{x^{\prime \prime} \rightarrow y^{\prime} \mid x \rightarrow y \in A(D)\right\} \cup\left\{x^{\prime} \rightarrow x^{\prime \prime} \mid x \in V(D)\right\}$. All arcs have capacity one. All arcs of the kind $w^{\prime} \rightarrow w^{\prime \prime}$, where $w \in W$, have lower bound one. All other arcs have lower bound zero.

It is easy to see that $D$ has the desired cycle subgraph if and only if $N$ has an integer valued feasible circulation. Furthermore, in the case when all capacities are one and only $|W| \leq n$ arcs have lower bounds different from zero, we can find such a circulation in time $O(\mathrm{~nm})$ (if one exists). For an explicit algorithm achieving this complexity see e.g. [1, page 195].

An easy consequence of Lemma 3.1 is the following:

Lemma 3.2 Let $D=(V(D), A(D))$ be a digraph on $n$ vertices and $m$ arcs, and subsets $R, S, T \subseteq V(D)$ (not necessarily disjoint). In time $O(\mathrm{~nm})$ we can either find a 1-pathcycle subgraph $P \cup C_{1} \cup \ldots \cup C_{k}$ which covers $T$ such that $P$ starts in $R$ and terminates in $S$ or show that such a subgraph does not exist.

Proof: Let $D^{\prime}$ be obtained from $D$ by adding a new vertex $x$ and all possible arcs from $x$ to vertices in $R$ as well as all possible arcs from vertices in $S$ to $x$. Now it is easy to see that $D$ has a 1-path-cycle subgraph with the required properties if and only if $D^{\prime}$ has a cycle subgraph which covers $T$. Thus the claim follows from Lemma 3.1. $\diamond$.

Note 3.3 In the rest of the paper the digraph $D$ being considered is a semicomplete multipartite digraph of order $n$ with colour classes $V_{1}, V_{2}, \ldots, V_{c}$; the colour class containing a vertex $x \in V(D)$ is denoted by $V_{\text {col }(x)}$, where col $(x)$ stands for the corresponding integer from $\{1,2, \ldots, c\}$.

Note that if $C, C^{\prime}$ are disjoint cycles in a semicomplete multipartite digraph $D$, then (up to switching the role of the two cycles) at least one of the following four cases apply (see Figure 3):

(A) Every vertex on $C$ has an arc to and from $C^{\prime}$.

(B) There exist vertices $x \in V(C), y \in V\left(C^{\prime}\right)$ such that $x \Rightarrow V\left(C^{\prime}\right)$ and $y \Rightarrow V(C)$, or $V\left(C^{\prime}\right) \Rightarrow x$ and $V(C) \Rightarrow y$.

(C) $C$ contains distinct vertices $x, y$ such that $x \Rightarrow V\left(C^{\prime}\right)$ and $V\left(C^{\prime}\right) \Rightarrow y$. 


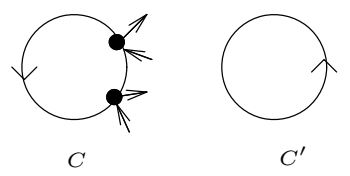

(A)

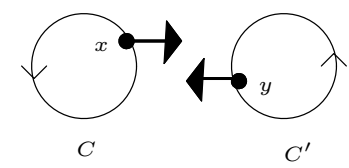

(B)

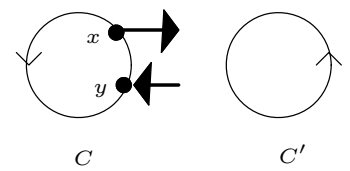

(C)

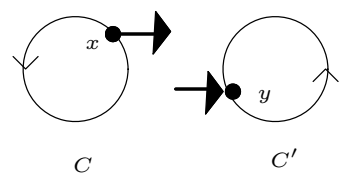

(D)

Figure 3: The four possible situations (up to switching the role of the two cycles or reversing all arcs) for arcs between two disjoint cycles in a semicomplete multipartite digraph. In (A) every vertex on $C$ has arcs to and from $C^{\prime}$. In (B)-(D) a fat arc indicates that all arcs go in the direction shown from or to the specified vertex (i.e. in (B) all $\operatorname{arcs}$ between $x$ and $C^{\prime}$ leave $x$ ).

(D) $C$ contains a vertex $x$ such that $x \Rightarrow V\left(C^{\prime}\right)$ and $C^{\prime}$ contains a vertex $y$ such that $V(C) \Rightarrow y$.

The following result was proved in [3]:

Theorem 3.4 If $D$ is a semicomplete multipartite digraph with disjoint cycles $C_{1}, C_{2}$ for which one of the alternatives (A)-(C) above holds, then in time $O\left(\left|V\left(C_{1}\right)\right| \times\left|V\left(C_{2}\right)\right|\right)$ one can find a cycle $C$ in $D$ with $V(C)=V\left(C_{1}\right) \cup V\left(C_{2}\right)$.

Since we shall use the existence of this algorithm a number of times in the rest of the paper, we give it a name, Merge. The algorithm Merge takes as input the two cycles $C_{1}, C_{2}$ and if one of the alternatives (A)-(C) holds, it replaces these with one cycle $C$ such that $V(C)=V\left(C_{1}\right) \cup V\left(C_{2}\right)$. If alternative (D) holds, then Merge leaves the cycles unchanged (note that this does not necessarily mean that there is no cycle $C$ as above).

Let us return to the situation in Theorem 3.4. In the case when only alternative (D) holds the third author [21] proved (as part of a stronger result on minimal factors in semicomplete multipartite digraphs, see Theorem 3.7 below) that if there are arcs in both directions between $C_{1}$ and $C_{2}$ then one can still merge the cycles into a cycle $C$ as above in the same time, unless the following holds for $i=1$ or $i=2$ : there exists a colur class $V_{\chi}$ of $D$ such that all $\operatorname{arcs} x \rightarrow y$ from $C_{3-i}$ to $C_{i}$ satisfy that $x^{+}, y^{-} \in V_{\chi}$. Recall that $x^{+}$is the successor of $x$ on $C_{3-i}$ and $y^{-}$is the predecessor of $y$ on $C_{i}$.

In order to formulate Theorem 3.7 we need the following very important definition $[21]$.

Definition 3.5 Let $D$ be a semicomplete multipartite digraph, let $X \subset V(D)$ and let $\mathcal{F}=C_{1} \cup C_{2} \cup \ldots \cup C_{t}$ be a cycle subgraph of $D . \mathcal{F}$ is called $X$-singular if every cycle of $\mathcal{F}$ contains a vertex from $X$, and either $t=1$, or $\mathcal{F}$ satisfies the following properties:

$(\alpha)$ The cycles in $\mathcal{F}$ are labeled such that $C_{i} \simeq C_{j}$ for every pair $i, j(1 \leq i<j \leq t)$ (i.e. only alternative (D) form Figure 3 holds for $C_{i}$ and $C_{j}$ ).

( $\beta$ ) There are colours $\chi_{R}\left(C_{i}\right) \in\{1,2, \ldots, c\}$ for $i=1, \ldots, t-1$ and $\chi_{L}\left(C_{j}\right) \in$ $\{1,2, \ldots, c\}$ for $j=2, \ldots, t$ such that every $\left(C_{j}, C_{i}\right)_{\mathcal{F}}$-path with $j>i$ starting 
at some vertex $z \in V\left(C_{j}\right)$ and terminating at some vertex $y \in V\left(C_{i}\right)$ satisfies that $y^{-} \in V_{\chi_{R}\left(C_{i}\right)} \cap X, z^{+} \in V_{\chi_{L}\left(C_{j}\right)} \cap X$.

$(\gamma)$ For every pair of cycles $C_{i}, C_{j}$ with $j>i$, if there exists a $\left(C_{j}, C_{i}\right)_{\mathcal{F}}$-path, then $\chi_{R}\left(C_{i}\right)=\chi_{L}\left(C_{j}\right)$.

See Figure 4 for an illustration of the definition. In the rest of this paper, the labeling of the cycles of a singular cycle subgraph (with at least two cycles) will be always made according to $(\alpha)$.

For future reference, we state a useful consequence of Definition 3.5.

Proposition 3.6 If $\mathcal{F}=C_{1} \cup C_{2} \cup \ldots \cup C_{t}$ is $X$-singular, then

$$
\begin{aligned}
& \text { 1. For all } i=1, \ldots, t-1: V\left(C_{i}\right) \cap V_{\chi_{R}\left(C_{i}\right)} \Rightarrow V\left(C_{i+1}\right) \cup \ldots \cup V\left(C_{t}\right) \\
& \text { 2. For all } j=2, \ldots, t: V\left(C_{1}\right) \cup \ldots \cup V\left(C_{j-1}\right) \Rightarrow V\left(C_{j}\right) \cap V_{\chi_{L}\left(C_{j}\right)} \text {. } \\
& \text { 3. For all } j=2, \ldots, t:\left(\bigcup_{i=1}^{j-1} V\left(C_{i}\right)\right) \cap V_{\chi_{L}\left(C_{j}\right)} \Rightarrow V\left(C_{j}\right) \text {. }
\end{aligned}
$$

Proof: We only prove 1. as the proofs of 2. and 3. are analogous. Suppose there is an arc $x \rightarrow y$ such that $x \in V\left(C_{j}\right), j \geq i+1$ and $y \in V\left(C_{i}\right) \cap V_{\chi_{R}\left(C_{i}\right)}$. Then it follows from Definition $3.5(\beta)$ that $y^{-} \in V_{\chi_{R}\left(C_{i}\right)}$, hence, $y^{-}$and $y$ belong to the same colour class; a contradiction.

The importance of singular cycle subgraphs is evident from the following theorem.

Theorem 3.7 [20, 21] Let $D$ be a semicomplete multipartite digraph and $X$ a specified subset of $V(D)$.

(a) If $D$ has a cycle subgraph covering $X$, then every cycle subgraph of $D$ covering $X$ with the minimum number of cycles is $X$-singular.

(b) There is an $O\left(n^{3}\right)$-time algorithm which given a cycle subgraph, $\mathcal{F}^{\prime}=C_{1}^{\prime} \cup C_{2}^{\prime} \cup$ $\ldots \cup C_{l}^{\prime}$, of $D$ covering $X$, constructs an $X$-singular cycle subgraph, $\mathcal{F}=C_{1} \cup$ $C_{2} \cup \ldots \cup C_{t}$ covering $X$ with the property that for every $i \in\{1,2, \ldots, l\}$ there is a $j \in\{1,2, \ldots, t\}$ such that $X \cap V\left(C_{i}^{\prime}\right) \subseteq V\left(C_{j}\right)$ (i.e. vertices from $X$ which are on one cycle in $\mathcal{F}$ remain on one cycle in $\left.\mathcal{F}^{\prime}\right)$.

Note 3.8 We shall use the name Singular for the algorithm of Theorem 3.7. Singular is an iterative algorithm. In each iteration, Singular starts with a cycle subgraph $\mathcal{H}$ covering $X$ and tries to merge two or more cycles of $\mathcal{H}$ into one cycle, using among other subroutines the algorithm Merge. When we merge some cycles, we may lose some vertices of the cycles but only some which are not in X. If Singular succeeds in merging some cycles, then we obtain a new cycle subgraph covering $X$ but with fewer cycles. The new cycle subgraph is the input for the next iteration. If however Singular has not succeeded to merge any set of two or more cycles in $\mathcal{H}$, then $\mathcal{H}$ is $X$-singular and Singular returns $\mathcal{H}$. 


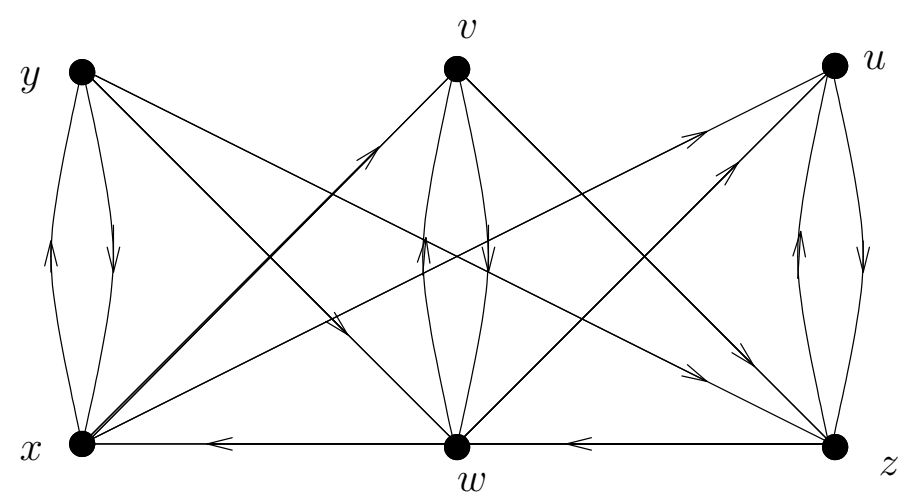

Figure 4: A semicomplete 3-partite digraph $H$ with a $X$-singular cycle subgraph $\mathcal{F}=$ $C_{1} \cup C_{2} \cup C_{3}$, where $C_{1}=x y x, C_{2}=v w v, C_{3}=u z u$. Here $X=V(H)$

If Singular is given, as input, the digraph $H$ in Figure 4 with $X=V(H)-\{v\}$ (instead of $X=V(H)$ ) and $\mathcal{F}=C_{1} \cup C_{2} \cup C_{3}$, as indicated in the figure, then it will return the cycle subgraph $\mathcal{F}^{\prime}=C_{1}^{\prime} \cup C_{2}^{\prime}$ with $C_{1}^{\prime}=x y w x, C_{2}^{\prime}=u z u$. Note that this cycle subgraph is $X$-singular and contains the minimum number of cycles among all cycle subgraphs covering $X$.

Even though being able to convert a given cycle subgraph $\mathcal{F}^{\prime}$ covering the set $X$ (which we wish to cover) into an $X$-singular cycle subgraph $\mathcal{F}$ is quite useful, it does not seem to make finding a possible cycle covering $X$ much easier. Consider for example the semicomplete multipartite digraph $H^{\prime}$ in Figure 5. Here $X=V\left(H^{\prime}\right)$ and the unique Hamiltonian cycle shows no nice patern with respect to the last cycle in $\mathcal{F}$. Clearly more complicated examples with even worse structure of a Hamiltonian cycle can be constructed.

In order to simplify the search for a more suitable structure on our cycle subgraph covering $X$, we introduce the notion of a leftshifted cycle subgraph in the next section. As we shall see in Lemma 4.2, if we combine the two properties of being $X$-singular and $X$-leftshifted, then we obtain a very special structure on the last cycle $C_{t}$ of our cycle subgraph. This property in turn allows us to look only for cycles covering $X$ with a very special structure in terms of vertices from the cycle $C_{t}$, the so-called $\mathcal{F}$-canonical cycles (see Lemma 5.2 and Figure 6). Once we have all this structure we will show in Theorem 5.3 that the desired cycle can be found using a recursive approach (via redefining $X$ ). The main obstruction - how to create an $X$-singular and $X$-leftshifted cycle subgraph covering $X$ - is handled in Section 6 .

\section{Leftshifted singular cycle subgraphs}

As pointed out in Section 3, we need some tool to transform a given $X$-singular cycle subgraph into one which can be used efficiently when searching for a cycle covering $X$. As we shall see in Lemma 4.2 and Lemma 5.2 the notion of leftshifted defined below is the desired tool for this. 


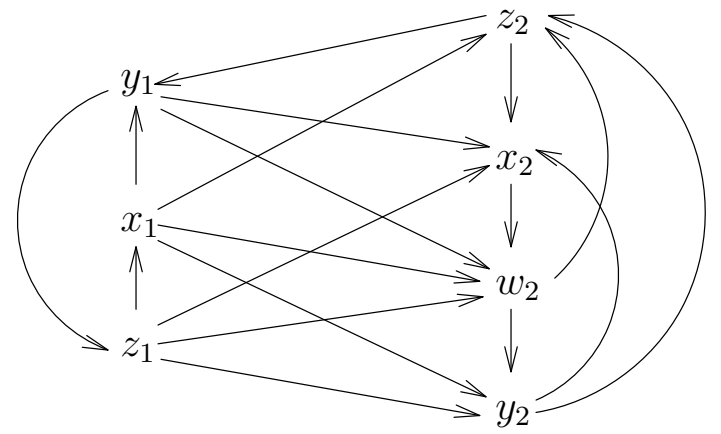

(a)

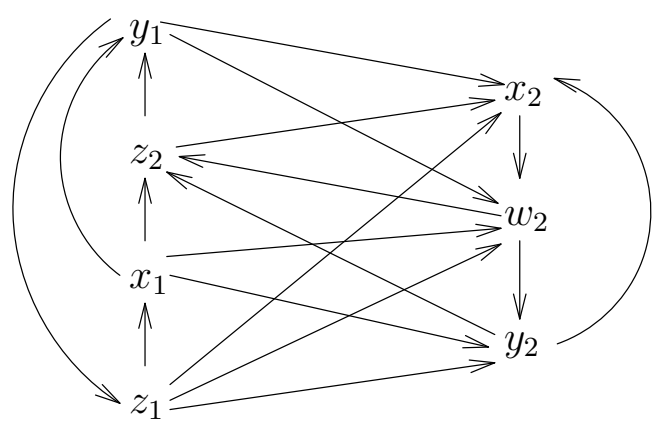

(b)

Figure 5: A semicomplete 4-partite digraph $H^{\prime}$. Let $X=V\left(H^{\prime}\right)$. Part (a) shows an $X$ singular cycle subgraph $\mathcal{F}=C \cup C^{\prime}$ with $C=x_{1} y_{1} z_{1} x_{1}, C^{\prime}=z_{2} x_{2} w_{2} y_{2} z_{2}$. The unique Hamiltonian cycle in $H$ is $y_{1} z_{1} x_{1} y_{2} x_{2} w_{2} z_{2} y_{1}$. Part (b) shows another cycle subgraph $\mathcal{F}^{\prime}=C_{1} \cup C_{2}$ with $C_{1}=z_{1} x_{1} z_{2} y_{1} z_{1}, C_{2}=x_{2} w_{2} y_{2} x_{2}$. Note that $\mathcal{F}^{\prime}$ is not $X$-singular, because $C_{1} \simeq C_{2}$, but $w_{2} \rightarrow z_{2}$ and $w_{2}^{+}$and $z_{2}^{-}$are adjacent.

Definition 4.1 A cycle subgraph $\mathcal{F}=C_{1} \cup C_{2} \cup \ldots \cup C_{t}$ is $X$-leftshifted if either $t=1$, or it has the following property for every choice of $i, j$ with $1 \leq i<j \leq t$ : There is no 1 path-cycle subgraph $\mathcal{F}^{\prime}=P \cup Z_{1} \cup \ldots \cup Z_{k}$ such that $X \cap V\left(C_{j}\right) \subseteq V\left(\mathcal{F}^{\prime}\right) \cap V(\mathcal{F}) \subseteq V\left(C_{j}\right)$, $P$ is a $(u, v)$-path and there exists $w \in V\left(C_{i}\right)$ such that $w \rightarrow u$ and $v \rightarrow w^{+}$.

Notice that if $\mathcal{F}$ is not $X$-leftshifted then, for some choice of $i, j$ with $1 \leq i<j \leq t$, there is a 1-path-cycle subgraph $\mathcal{F}^{\prime}$ with $P$ as its path, such that the cycle $C_{i}$ can be replaced by a cycle $C_{i}^{\prime}$ where $V\left(C_{i}\right) \cup V(P)=V\left(C_{i}^{\prime}\right)$ and $C_{j}$ can be replaced by the cycle subgraph $\mathcal{F}^{\prime}-P$ such that $C_{i}^{\prime} \cup\left(\mathcal{F}-C_{i} \cup C_{j}\right) \cup\left(\mathcal{F}^{\prime}-P\right)$ is a new cycle subgraph which covers $V(\mathcal{F}) \cap X$. For example, if we let $X=V(H)-v$, then with respect to the ordering $C_{1}, C_{2}, C_{3}$, the cycle subgraph $\mathcal{F}$ in Figure 4 is not $X$-leftshifted since the vertex $w$ can be inserted in the cycle $x y x$ while leaving out the non-important vertex $v$. Similarly, with the ordering $C, C^{\prime}$ the cycle subgraph $\mathcal{F}$ in Figure 5 is also not $X$-leftshifted, as the vertex $z_{2}$ can be inserted in $C$ while leaving the cycle $C_{2}$.

The following technical lemma plays an important role in our algorithm in Section 5 as it forms the basis for Lemma 5.2.

Lemma 4.2 Let $X$ be a subset of $V(D)$, and let $\mathcal{F}=C_{1} \cup \ldots \cup C_{t}$, $t \geq 2$ be a $X$ leftshifted $X$-singular cycle subgraph which covers $X$. Let also $R=C_{1} \cup \ldots \cup C_{t-1}$ and $W=\left\{w \in V\left(C_{t}\right): \exists\left(C_{t}, R\right)\right.$-path starting at $w$ in $\left.D\right\}$. Suppose $W \neq \emptyset$. There exists a non-empty set $S \subset V\left(C_{t}\right)$ with the properties below:

$$
\begin{gathered}
S \subseteq V_{\chi_{L}\left(C_{t}\right)} \cap X \cap V\left(C_{t}\right) \\
S \Rightarrow V\left(C_{t}\right)-S^{-}
\end{gathered}
$$




$$
W \subseteq S^{-},
$$

for every $u \in V(D)-\left(V(\mathcal{F}) \cup V_{\chi_{L}\left(C_{t}\right)}\right)$, either $S \Rightarrow$ u or $D-V(R)$ has no $\left(C_{t}, u\right)$-path.

Proof: For every $w \in W$ we define the following: $S_{0}^{w}=\emptyset, S_{1}^{w}=\left\{w^{+}\right\}$and $S_{i}^{w}=$ $\left\{r \in V\left(C_{t}\right): \exists q \in S_{i-1}^{w}\right.$ such that $\left.r^{-} \rightarrow q\right\}$ for $i=2,3, \ldots,\left|V\left(C_{t}\right)\right|$. Observe that there exists $n_{w}(w \in W)$, such that $S_{0}^{w} \subset S_{1}^{w} \subset S_{2}^{w} \subset \ldots \subset S_{n_{w}}^{w}=S_{n_{w}+1}^{w}=\ldots=S_{\left|V\left(C_{t}\right)\right|}^{w}$. Let $S^{w}=S_{n_{w}}^{w}\left(=S_{\left|V\left(C_{t}\right)\right|}^{w}\right)$ and $S=\bigcup_{w \in W} S^{w}$. Let

$$
T^{\prime}=\left\{v \in V\left(C_{t}\right): \exists q \in V(D)-V(\mathcal{F})-V_{\chi_{L}\left(C_{t}\right)} \text { such that }\left(V_{\chi_{L}\left(C_{t}\right)} \cap V(R)\right) \Rightarrow q \rightarrow v\right\}
$$

and

$$
T=\left\{v \in V\left(C_{t}\right): v \notin V_{\chi_{L}\left(C_{t}\right)} \cap X\right\} \cup T^{\prime} .
$$

Claim: $T \cap S=\emptyset$

Proof of claim: Suppose that this is false, and let $w \in W$ be chosen such that $T \cap S^{w} \neq \emptyset$. We can now find a sequence of distinct vertices $x_{1}, x_{2}, \ldots, x_{a}\left(x_{i} \in\right.$ $\left.S_{i}^{w}-S_{i-1}^{w}, i=1, \ldots, a, a \geq 1\right)$, such that $x_{i+1}^{-} \rightarrow x_{i}$ and $x_{i} \notin T$ for $i=1,2, \ldots, a-1$ and $x_{a} \in T$.

A spanning 1-path-cycle subgraph $P \cup Z_{1} \cup \ldots \cup Z_{c}$ of $D\left\langle V\left(C_{t}\right)\right\rangle$ such that the path $P$ starts in $T$ and terminates in $W$ can be constructed as follows: start with the cycle $C_{t}$, delete the $\operatorname{arcs} x_{i}^{-} \rightarrow x_{i}, i=1,2, \ldots, a$ and add the $\operatorname{arcs} x_{i}^{-} \rightarrow x_{i-1}, i=2,3, \ldots, a$. In the digraph obtained in this way all vertices except $x_{1}^{-}$and $x_{a}$ have in- and out-degree one, $x_{1}^{-}$has out-degree zero and in-degree one and $x_{a}$ has in-degree zero and out-degree one. Thus the digraph described is a spanning 1-path-cycle subgraph of $D\left\langle V\left(C_{t}\right)\right\rangle$ with the path $P$ starting in $x_{a} \in T$ and ending in $x_{1}^{-}=w \in W$.

Let

$$
\begin{gathered}
U=\left\{u \in V(D)-V(R)-V_{\chi_{L}\left(C_{t}\right)}: V_{\chi_{L}\left(C_{t}\right)} \cap V(R) \Rightarrow u\right\}, \\
Z=\left\{z \in V(D)-V(R):\left(V_{\chi_{L}\left(C_{t}\right)} \cap V(R)\right)^{+} \not \Rightarrow z\right\},
\end{gathered}
$$

Let $P=p_{1} p_{2} \ldots p_{b}$. Since $p_{b} \in W$ there is a $\left(C_{t}, R\right)$-path, $U^{\prime}=u_{1}^{\prime} u_{2}^{\prime} \ldots u_{d}^{\prime}$, with $p_{b}=$ $u_{1}^{\prime}$. By the definition of a $\left(C_{t}, R\right)$-path, $u_{d-1}^{\prime} \in V(D)-V(R)$ and $\left(V_{\chi_{L}\left(C_{t}\right)} \cap R\right)^{+} \nRightarrow u_{d-1}^{\prime}$ (since $u_{d}^{\prime} \in\left(V_{\chi_{L}\left(C_{t}\right)} \cap R\right)^{+}$by Definition 3.5 and the fact that $\mathcal{F}$ is $X$-singular), which implies that $u_{d-1}^{\prime} \in Z$. Now consider the following possibilities:

If $p_{1} \notin V_{\chi_{L}\left(C_{t}\right)}$, then $p_{1} \in U$ by Proposition $3.6(3)$ and $P^{\prime}=P U^{\prime}\left[u_{1}^{\prime}, u_{d-1}^{\prime}\right]$ is a path from $U$ to $Z$.

If $p_{1} \in V_{\chi_{L}\left(C_{t}\right)}$ and $p_{1} \notin X$, then $p_{2} \in U$, by Proposition $3.6(3)$ and $P^{\prime}=P\left[p_{2}, p_{b}\right] U^{\prime}\left[u_{1}^{\prime}, u_{d-1}^{\prime}\right]$ is a path from $U$ to $Z$ (observe that $b \geq 2$, since $p_{b} \notin V_{\chi_{L}\left(C_{t}\right)}$ because $W \cap V_{\chi_{L}}\left(C_{t}\right)=\emptyset$ by Proposition $\left.3.6(2)\right)$.

If $p_{1} \in V_{\chi_{L}\left(C_{t}\right)} \cap X$, then, by the definition of $T, p_{1} \in T^{\prime}$ and there is a vertex $q \in$ $V(D)-V(\mathcal{F})-V_{\chi_{L}\left(C_{t}\right)}$ such that $\left(V_{\chi_{L}\left(C_{t}\right)} \cap R\right) \Rightarrow q \rightarrow p_{1}$, thus $q \in U$ and $P^{\prime}=$ $q P U^{\prime}\left[u_{1}^{\prime}, u_{d-1}^{\prime}\right]$ is a path from $U$ to $Z$. 
Observe that $P^{\prime} \cup Z_{1} \cup \ldots \cup Z_{c}$ is a 1-path-cycle subgraph in $D-V(R)$ which covers $V\left(C_{t}\right) \cap X$ and whose path $P^{\prime}$ starts in $U$ and terminates in $Z$. By the definitions of $U$ and $Z$, there exists $y \in V(R)$ such that $y$ dominates the first vertex of $P^{\prime}$ and $y^{+}$ is dominated by the last vertex of $P^{\prime}$. Thus, the existence of $P^{\prime} \cup Z_{1} \cup \ldots \cup Z_{c}$ is a contradiction against the assumption that $\mathcal{F}$ is leftshifted. This concludes the proof of the claim.

We will now prove properties (1), (2), (3) and (4). Since $S \cap T=\emptyset$ we get $S \subseteq$ $V_{\chi_{L}\left(C_{t}\right)} \cap X \cap V\left(C_{t}\right)$, which proves (1). From the definition of $S$ we get that $S \Rightarrow V\left(C_{t}\right)-$ $S^{-}$and $W \subseteq S^{-}$, which proves (2) and (3). To prove (4) suppose $u \in V(D)-V(\mathcal{F})-$ $V_{\chi_{L}\left(C_{t}\right)}$ is a vertex such that $S \not \nexists u$. Let $s \in S$ be arbitrary with $u \rightarrow s$. We must have $\left(V_{\chi_{L}\left(C_{t}\right)} \cap R\right) \nRightarrow u$, since otherwise $s \in T^{\prime}$ which is a contradiction by the claim. Let $q \in\left(V_{\chi_{L}\left(C_{t}\right)} \cap R\right)$ be arbitrary such that $u \rightarrow q$. If there is a $\left(C_{t}, u\right)$-path, $P$, in $D-V(R)$, then $P q$ is a $\left(C_{t}, R\right)$-path, $p_{1} p_{2} \ldots p_{l} u q$, with $q^{-} \notin V_{\chi_{L}}\left(C_{t}\right)$ which is a contradiction against the the fact that $\mathcal{F}$ is singular, by Definition 3.5 (the definition of $V_{\chi_{L}\left(C_{t}\right)}$ ). Therefore, if $S \not \nexists u$, then there is no $\left(C_{t}, u\right)$-path in $D-V(R)$. This proves (4) and completes the proof of the lemma.

Note 4.3 In the rest of this paper we distinguish a subset $X$ of $V(D)$; let the vertices in $X$ be called $X$-vertices and make the following assumptions about $X$ if $\bar{X}=V(D)-X \neq$ $\emptyset$ :

$$
\begin{gathered}
|\bar{X}| \geq 2 \\
X \rightarrow \bar{X} \\
\bar{X} \subseteq V_{\chi} \text { for some colour } \chi \in\{1,2, \ldots, c\}
\end{gathered}
$$

There is a pair of vertices $x_{\alpha}, x_{\beta}$ in $X$ such that $x_{\alpha} \in V_{\operatorname{col}(\bar{X})},\left|V_{\operatorname{col}\left(x_{\beta}\right)}\right|=1$,

and

$$
X-\left\{x_{\beta}\right\} \Rightarrow x_{\beta} \Rightarrow \bar{X}, X-\left\{x_{\beta}\right\} \Rightarrow x_{\alpha}
$$

Notice, in particular, that by (7), every cycle of $D$ contains a vertex from $X$.

For future reference we state two easy consequences of these assumptions.

Lemma 4.4 If $\mathcal{F}=C_{1} \cup C_{2} \cup \ldots \cup C_{t}$ is an $X$-singular cycle subgraph of $D$, then $V\left(C_{1} \cup C_{2} \cup \ldots \cup C_{t-1}\right) \subseteq X$.

Proof: Suppose there exists a vertex $q \in V\left(C_{1} \cup C_{2} \cup \ldots \cup C_{t-1}\right) \cap \bar{X}$. Let $x \in X \cap V\left(C_{t}\right)$ be chosen such that $\operatorname{col}(x) \neq \operatorname{col}(q)$ (this is possible since $\bar{X} \subseteq V_{\chi}$ for some colour $\chi$ ). Then $x \rightarrow q$, since $X \rightarrow \bar{X}$ (see Note 4.3). By Definition 3.5, $x^{+}$, $q^{-} \in X \cap V_{\chi_{L}\left(C_{t}\right)}$. Since $\operatorname{col}\left(x^{+}\right)=\operatorname{col}\left(q^{-}\right)$and $x^{+} \in X, x^{+} \rightarrow q$. By Definition 3.5, we get $x^{++} \in V_{\chi_{L}\left(C_{t}\right)}$. Thus we conclude that both $x^{+}$and $x^{++}$are in $V_{\chi_{L}\left(C_{t}\right)}$; a contradiction. $\diamond$. 
Lemma 4.5 If $\bar{X} \neq \emptyset$ and $\mathcal{F}=C_{1} \cup \ldots \cup C_{t}$ is an $X$-singular cycle subgraph which covers $X$, with $R=C_{1} \cup \ldots \cup C_{t-1}$, then the following holds:

The arc $x_{\alpha} \rightarrow x_{\beta}$ is an arc of $C_{t}$

$$
V(R) \Rightarrow V(D)-V(\mathcal{F})
$$

Proof: As $x_{\beta}$ is the only out-neighbour of $x_{\alpha}$, by (8) and (9), the arc $x_{\alpha} \rightarrow x_{\beta}$ belongs to a cycle in $\mathcal{F}$ and since the only out-neighbours of $x_{\beta}$ are in $\bar{X}$ it follows from Lemma 4.4 that $x_{\alpha} \rightarrow x_{\beta}$ is an arc of $C_{t}$. Assume that (11) is false and, thus, there exist $y \in V(D)-V(\mathcal{F}), z \in R$ such that $y \rightarrow z$. As $y$ is not in $X, x_{\beta} \rightarrow y$. Hence, $x_{\beta} y z$ is a $\left(C_{t}, R\right)$-path. Thus, by Lemma $4.2, x_{\beta}^{+} \in X$; a contradiction.

Our goal is to describe a polynomial algorithm for deciding whether $D$ has a cycle covering $X$. For this we need to be able to make a given cycle subgraph covering $X$ $X$-singular and $X$-leftshifted. The highly technical proof of the following crucial result is postponed to Section 6:

Theorem 4.6 Given any cycle subgraph in $D$ which covers $X$, in $O\left(n^{6}\right)$ time, we can find an $X$-singular $X$-leftshifted cycle subgraph, $\mathcal{F}=C_{1} \cup C_{2} \cup \ldots \cup C_{t}$ which covers $X$.

\section{The main algorithm}

Below, we shall use the notation adopted in the formulation of Lemma 4.2.

In this section $\mathcal{F}=C_{1} \cup \ldots \cup C_{t}(t \geq 2)$ denotes an $X$-singular $X$-leftshifted cycle subgraph of $D$ which covers $X, R=C_{1} \cup \ldots \cup C_{t-1}$ and $S$ is the set determined in Lemma 4.2.

Definition 5.1 A cycle $C$ in $D$ is called $\mathcal{F}$-canonical if there exists a subset $L=$ $\left\{v_{1}, \ldots, v_{l}\right\}$ of $S^{-}$such that $C_{t}\left[v_{i}, v_{i+1}\right]$ contains no vertices from $L-\left\{v_{i}, v_{i+1}\right\}$ for all $i=1, \ldots, l$ and with the property that $C$ has the form

$$
C=C_{t}\left[v_{l}^{+}, v_{1}\right] T_{1} C_{t}\left[v_{1}^{+}, v_{2}\right] T_{2} \ldots T_{l-1} C_{t}\left[v_{l-1}^{+}, v_{l}\right] T_{l} v_{l}^{+} \text {, }
$$

where $T_{i}$ is a path in $D\langle R\rangle$ for all $i=1,2, \ldots, l$ (see Figure 6 ).

The following lemma shows the usefulness of $\mathcal{F}$-canonical cycles and also justifies the definition of an $X$-leftshifted cycle subgraph.

Lemma 5.2 Suppose that $D$ contains a cycle covering $X$, and that $\mathcal{F}=C_{1} \cup \ldots \cup C_{t}$ $(t \geq 2)$ is an $X$-leftshifted $X$-singular cycle subgraph of $D$ covering $X$. Then $D$ has an $\mathcal{F}$-canonical cycle covering $X$.

Proof: Let $Q=V(D)-V(\mathcal{F})$ and $V^{*}=V_{\chi_{L}\left(C_{t}\right)}$. Let $Z$ be a cycle of $D$ covering $X$ and let $Z\langle D-V(R)\rangle=P_{1} \cup P_{2} \cup \ldots \cup P_{l}$, where $P_{1}, \ldots, P_{l}$ are pairwise vertex disjoint paths. Recall that, by Note 4.3 , 


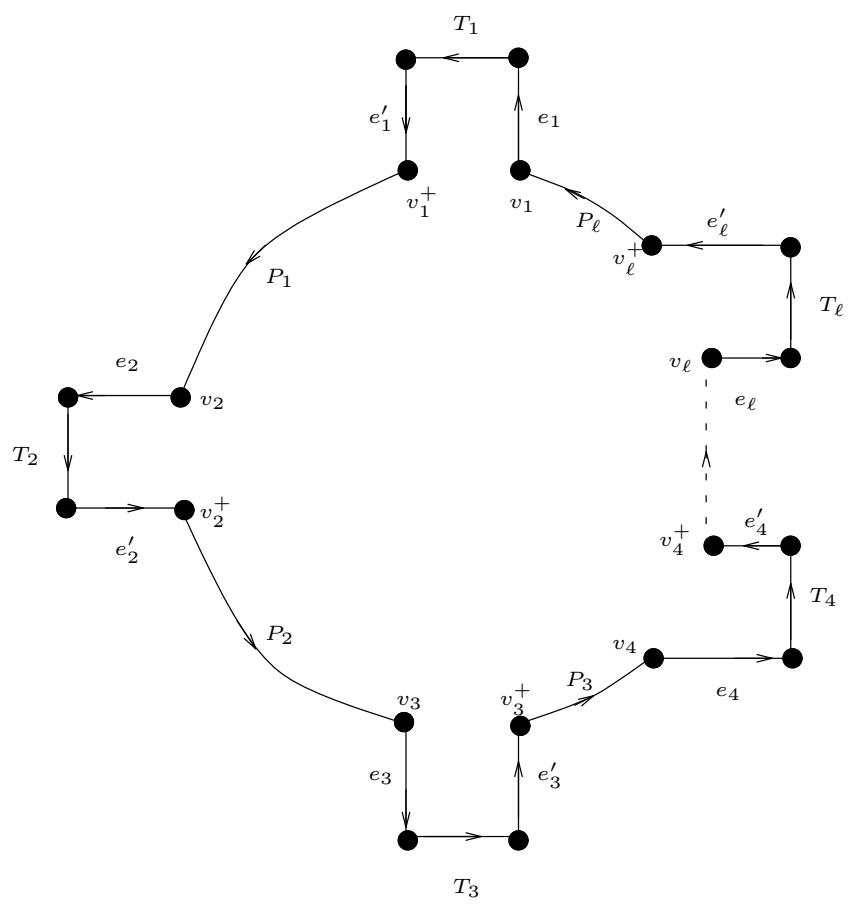

Figure 6: An example of an $\mathcal{F}$-canonical cycle for some $X$-singular $X$-leftshifted cycle subgraph $\mathcal{F}=C_{1} \cup \ldots \cup C_{t}$ which covers $X$. Each $P_{i}$ is of the form $P_{i}=C_{t}\left[v_{i}^{+}, v_{i+1}\right]$, all $e_{i}, e_{i}^{\prime}$ denote arcs and each $T_{i}$ is a path in $D\langle R\rangle$

all vertices of $Q$ are from the same colour class $V_{\operatorname{col}(\bar{X})}$.

By (11), the last vertex $v_{i}$ of $P_{i}$ is from $C_{t}$ and the successor $w_{i}$ of $v_{i}$ on $Z$ must be from $R$. Therefore, by the definition of $W$ in Lemma 4.2 and by (3), $v_{i} \in S^{-}$. Thus, since $|S|=\left|S^{-}\right|$and $S \subseteq X$, there are $l$ vertices from $S$ whose predecessors on $Z$ are not from $S^{-}$. Suppose $s \in S$ on $P_{i}$ and is not the first vertex of $P_{i}$. We show that the predecessor $u$ of $s$ on $P_{i}$ is in $S^{-}$. Assume that $u \notin S^{-}$. By (2), $u \in Q$. By (1) and (4), there is no $\left(C_{t}, u\right)$-path in $D-V(R)$. However, by $(6), s \rightarrow u$; a contradiction. This implies that the first vertex $u_{i}$ of $P_{i}$ is in $S$ for each $i=1,2, \ldots, l$. Observe that by the definition of the paths $P_{1}, \ldots, P_{l}$, the predecessor $r_{i}$ of $u_{i}$ on $Z$ is from $R$.

Without loss of generality, assume that $v_{1}, v_{2}, \ldots, v_{l}$ are labeled such that the subpath $C_{t}\left[v_{i}, v_{i+1}\right]$ contains no vertices from the set $\left\{v_{1}, \ldots, v_{l}\right\}-\left\{v_{i}, v_{i+1}\right\}$ for all $i=1, \ldots, l$. Let $v_{i}^{+}$be the successor of $v_{i}$ on $C_{t}$. Since $r_{i} \rightarrow u_{i}$ and $u_{i} \in S \subseteq V^{*}\left(\right.$ see (1)), $r_{i}$ is not in $V^{*}$. As $v_{i} \in S^{-}, v_{i}^{+} \in S \subseteq V^{*}$ and thus $r_{i} \rightarrow v_{j}^{+}$for all $i$ and $j$ by Proposition 3.6 (2). Replace the subpaths $P_{i}\left[u_{i}, v_{i}\right]$ with the subpaths $C_{t}\left[v_{i-1}^{+}, v_{i}\right]$ in $Z$ for all $i=1,2, \ldots, l$. By the arguments above, the subgraph obtained after these substitutions is an $\mathcal{F}$-canonical cycle in $D$ covering $X$.

$\diamond$.

Theorem 5.3 We can verify whether $D$ contains a cycle covering $X$ (and construct one if it exists) in time $O\left(n^{7}\right)$. 
Proof: A short scheme of the algorithm is as follows: Using Lemma 3.1 try to construct a cycle subgraph $\mathcal{F}^{\prime}$ of $D$ covering $X$. If none exists, then $D$ has no cycle covering $X$. Otherwise, applying Theorem 4.6 we can transform $\mathcal{F}^{\prime}$ into an $X$-leftshifted $X$ singular cycle subgraph $\mathcal{F}=C_{1} \cup \ldots \cup C_{t}$ using the algorithms Singular and Leftshift (see the definition of Leftshift in Section 6). If $t=1$, we are done. Otherwise, using Lemma 5.2, we reduce the problem of finding a cycle in $D$ covering $X$ into the problem of finding a cycle covering a new set $X^{*}$ in a new semicomplete multipartite digraph $D^{*}$ with fewer vertices. As the reduction takes $O\left(n^{6}\right)$ time (see Theorem 4.6) and we perform $O(n)$ reductions, the complexity of our algorithm is $O\left(n^{7}\right)$.

Note that by Definition 5.1 and Lemma 5.2 we may assume that $\mathcal{F}$ is a spanning cycle subgraph, since no $\mathcal{F}$-canonical cycle contains vertices from $V(D)-V(\mathcal{F})$. Let $V^{*}=V_{\chi_{L}\left(C_{t}\right)}$ and let $Z=\left\{z \in S^{-}: R \nRightarrow z\right\}$. If $Z$ is empty, then by (3) there is no path from $C_{t}$ to $R$, which means that $D$ has no cycle covering $X$ (recall that every cycle contains a vertex from $X$ (see Note 4.3)). In the rest of the proof we assume that $Z$ is not empty.

Case 1: $Z=\{z\}$. Set $V\left(D^{*}\right)=V(R) \cup\{z\}, A\left(D^{*}\right)=A(D\langle R\rangle) \cup\{z \rightarrow r: r \in$ $V(R), z \rightarrow r$ in $D\} \cup\left\{r \rightarrow z: \quad r \in V(R)-V^{*}\right\}$ and $X^{*}=V(R) \cup\{z\}$. Suppose $D$ has a cycle covering $X$. Then, by Lemma 5.2, $D$ has an $\mathcal{F}$-canonical cycle $C$ covering $X$. Since $|Z|=1, C$ must contain the path $C_{t}\left[z^{+}, z\right]$ and by the definition of an $\mathcal{F}_{-}$ canonical cycle, the predecessor $r$ of $z^{+}$on $C$ does not belong to $V^{*}$. Hence we get a cycle containing $X^{*}$ in $D^{*}$ just by replacing the path $r C_{t}\left[z^{+}, z\right]$ in $C$ by the $\operatorname{arc} r \rightarrow z$. Conversely, if $C^{*}$ is a cycle covering $X^{*}$ in $D^{*}$, then the predecessor $r^{*}$ of $z$ on $C^{*}$ does not belong to $V^{*}$ and hence we can convert $C^{*}$ to a cycle covering $X$ in $D$ by substituting the path $r^{*} C_{t}\left[z^{+}, z\right]$ for the $\operatorname{arc} r^{*} \rightarrow z$. Thus we have shown that $D^{*}$ has a cycle covering $X^{*}$ if and only if $D$ has a cycle covering $X$. Clearly, $|V(D)|>\left|V\left(D^{*}\right)\right|$.

Case 2: $|Z| \geq 2$. First construct a new digraph $D^{\prime}$ as follows: Set $V\left(D^{\prime}\right)=$ $V(R) \cup Z \cup\left\{a^{\prime}, b^{\prime}\right\}$, where $a^{\prime}$ and $b^{\prime}$ are new vertices, $A\left(D^{\prime}\right)=A(D\langle R\rangle) \cup\{z \rightarrow r: z \in$ $Z, r \in V(R), z \rightarrow r$ in $D\} \cup\left\{r \rightarrow z: r \in V(R)-V^{*}, z \in Z\right\} \cup\left\{a^{\prime} \rightarrow b^{\prime}\right\} \cup\left\{r \rightarrow b^{\prime}: r \in\right.$ $V(R)\} \cup\left\{r \rightarrow a^{\prime}: r \in V(R)-V^{*}\right\} \cup\left\{b^{\prime} \rightarrow z: z \in Z\right\}, X^{\prime}=V(R) \cup\left\{a^{\prime}, b^{\prime}\right\}$. Observe that in $D^{\prime}$ the vertex $a^{\prime}$ and all vertices of $Z$ are in the colour class $V^{*}$ (compare with Note $4.3, a^{\prime}, b^{\prime}$ play the role of $x_{\alpha}$ and $x_{\beta}$ ).

Claim : $D$ contains a cycle covering $X$ if and only if $D^{\prime}$ has a cycle covering $X^{\prime}$.

Proof of claim: Suppose that $D$ has a cycle covering $X$. Then, by Lemma 5.2, $D$ contains a $\mathcal{F}$-canonical cycle $C$ covering $X$. Let $C$ have the form given in (12). Transform $C$ into a cycle $C^{\prime}$ in $D^{\prime}$ covering $X^{\prime}$ as follows: For every $i=1,2, \ldots, l$, replace the path $C_{t}\left[v_{i-1}^{+}, v_{i}\right] T_{i}$ in $C$ with the path $v_{i} T_{i}$. By the definition of an $\mathcal{F}_{-}$ canonical cycle, the terminal vertex $r_{i}$ in $T_{i}$ is not in $V^{*}$ (as $v_{i}^{+} \in V^{*}$ ). Thus, by the definition of $D^{\prime}, r_{i-1} \rightarrow v_{i}$ in $D^{\prime}$ since $v_{i} \in Z$ (for all $\left.i=1,2, \ldots, l\right)$. Therefore, after all substitutions above, we obtain a cycle. Since $r_{i-1} \rightarrow a^{\prime}$ and $b^{\prime} \rightarrow v_{i}$ in $D^{\prime}$ for $i=1,2, \ldots, l$, the arc $a^{\prime} \rightarrow b^{\prime}$ can be inserted into this cycle to form a cycle $C^{\prime}$ covering $X^{\prime}$.

Suppose now that $D^{\prime}$ has a cycle $C^{\prime}$ covering $X^{\prime}$. Since $b^{\prime}$ is the only out-neighbour 
of $a^{\prime}$, the arc $a^{\prime} \rightarrow b^{\prime}$ is on $C^{\prime}$. Thus, since $b^{\prime}$ only dominates vertices in $Z, Z^{\prime}=$ $Z \cap V\left(C^{\prime}\right) \neq \emptyset$. Let $z \in Z$ be the successor of $b^{\prime}$ on $C^{\prime}$ and let $r$ be the predecessor of $a^{\prime}$ on $C^{\prime}$. By the definition of $D^{\prime}, r \rightarrow z$ in $D^{\prime}$. Set $C^{\prime \prime}=C^{\prime}[z, r] z$.

Let $Z^{\prime}=\left\{z_{1}, z_{2}, \ldots, z_{l}\right\}$, where the vertices $z_{1}, \ldots, z_{l}$ are labeled such that no vertex from $\left\{z_{1}, z_{2}, \ldots, z_{l}\right\}-\left\{z_{i}, z_{i+1}\right\}$ is on $C_{t}\left[z_{i}, z_{i+1}\right]$ for all $i=1,2, \ldots, l$. Let $r_{i}$ be the predecessor of $z_{i}$ on $C^{\prime \prime}$. By the definition of $D^{\prime}, r_{i} \in V(R)-V^{*}$. Thus, by Proposition 3.6 (2) and (1), $r_{i} \rightarrow z_{i-1}^{+}$in $D$ (recall that $z_{i-1}^{+}$is the successor of $z_{i-1}$ on $C_{t}$ ). Replace every $\operatorname{arc} r_{i} \rightarrow z_{i}$ in $C^{\prime \prime}$ with the path $r_{i} C_{t}\left[z_{i-1}^{+}, z_{i}\right]$. Clearly, the resulting cycle in $D$ covers $X$. Note that above we actually proved how to construct the cycle $C$ from $C^{\prime}$. This completes the proof of the claim.

If $D^{\prime}$ contains fewer vertices than $D$, then set $D^{*}=D^{\prime}$ and $X^{*}=X^{\prime}$. Assume now that $\left|V\left(D^{\prime}\right)\right| \geq|V(D)|$. Observe that then $|Z| \geq 2$ implies that $V\left(C_{t}\right)=S \cup S^{-}$and $\left|V\left(C_{t}\right)\right|=4$, thus we must have $\left|V\left(D^{\prime}\right)\right|=|V(D)|$.

Suppose that $\bar{X} \neq \emptyset$. Then, by Lemma 4.5 , the $\operatorname{arc} x_{\alpha} \rightarrow x_{\beta}$ is on $C_{t}$. Note that $x_{\beta} \notin S^{-}$, since $S \subset X$ and $X-\left\{x_{\beta}\right\} \Rightarrow x_{\beta}$. Hence by the remark above $\left(V\left(C_{t}\right)=S \cup S^{-}\right)$, we must have $x_{\beta} \in S$. However, then we would get $|Z| \leq 1$ (by (1), (8) and Definition $3.5(\beta))$, a contradiction. Thus we must have $\bar{X}=\emptyset$. Apply the construction of $D^{\prime}, X^{\prime}$ once more to $D^{\prime}$ and $X^{\prime}$ instead of $D$ and $X$ starting, from a new $X^{\prime}$-leftshifted $X^{\prime}$-singular cycle subgraph of $D^{\prime}$ (if there is no cycle subgraph covering $X^{\prime}$ in $D^{\prime}$, then by the claim, $D$ has no cycle covering $X)$. Finally, set $D^{*}=\left(D^{\prime}\right)^{\prime}, X^{*}=\left(X^{\prime}\right)^{\prime}$. Observe that, by construction, $V\left(D^{\prime}\right)-X^{\prime} \neq \emptyset$ and hence $|V(D)|=\left|V\left(D^{\prime}\right)\right|>\left|V\left(D^{*}\right)\right|$. Furthermore, by the arguments above, $D$ has a cycle covering $X$ if and only if $D^{*}$ has a cycle covering $X^{*}$.

This completes the description of our algorithm and the proof of its correctness. $\diamond$.

Clearly, Theorem 5.3 implies our main goal:

Corollary 5.4 The Hamiltonian cycle problem is solvable in polynomial time for semicomplete multipartite digraphs.

\section{Constructing leftshifted singular cycle subgraphs}

The purpose of this section is to prove Theorem 4.6.

Lemma 6.1 Suppose $\bar{X} \neq \emptyset$ and that $C$ and $C^{\prime}$ are disjoint cycles such that the arc $x_{\alpha} \rightarrow x_{\beta}$ is on $C$ and $C^{\prime}$ contains a vertex $z \in \bar{X}$, then there exists a cycle $C^{*}$ with $V\left(C^{*}\right)=V(C) \cup V\left(C^{\prime}\right)$.

Proof: Since $x_{\beta}$ only dominates vertices in $\bar{X}$, its sucessor $u$ on $C$ belongs to $\bar{X}$. By Note $4.3, x_{\beta}$ dominates $z$ and the predecessor $z^{-}$of $z$ on $C^{\prime}$ dominates $u$, implying that $C\left[u, x_{\beta}\right] C^{\prime}\left[z, z^{-}\right]$is the desired cycle.

$\diamond$.

Lemma 6.2 Let $\mathcal{F}=C_{1} \cup C_{2} \cup \ldots \cup C_{t}$ be an $X$-singular cycle subgraph and let $B$ be a cycle in $D-V(\mathcal{F})$. Let $A \subseteq \bar{X}-V(\mathcal{F} \cup B), D^{\prime}=D\langle V(\mathcal{F} \cup B) \cup A\rangle$ and $X^{\prime}=X \cap V\left(D^{\prime}\right)$. Suppose that every cycle $C_{i} \in \mathcal{F}(1 \leq i \leq t)$ has a vertex $q_{i} \in V\left(C_{i}\right) \cap X$, such that 
$q_{i} \Rightarrow V\left(C_{i+1} \cup C_{i+2} \cup \ldots \cup C_{t} \cup B\right)$. Then, in $O\left(n^{4}\right)$ time, we can find an $X$-singular cycle subgraph, $\mathcal{F}^{\prime}=Z_{1} \cup Z_{2} \cup \ldots \cup Z_{l} \cup B^{\prime}$ of $D^{\prime}$ covering $X^{\prime}$, where $l \in\{0,1, \ldots, t\}, B^{\prime}$ is a cycle with $V\left(C_{l+1} \cup C_{l+2} \cup \ldots \cup C_{t} \cup B\right) \cap X \subseteq V\left(B^{\prime}\right)$ and $Z_{i}=C_{i}$ for $i=1,2, \ldots, l$.

Proof: We first describe a procedure Left-B-growth which transforms $\mathcal{F}$ into $\mathcal{F}^{\prime}$ defined above. Then we shall prove the correctness of Left-B-growth and determine its complexity. Let $\mathcal{G}$ be a cycle subgraph of $D$. In the procedure we apply Singular (see Note 3.8) to $\mathcal{G}$ in the digraph $D\langle\mathcal{G}\rangle$ in order to find an $X$-singular subgraph $\mathcal{L}$ of $D\langle\mathcal{G}\rangle$ which covers $X \cap V(D\langle\mathcal{G}\rangle)$. We denote this as follows: $\mathcal{L}:=\operatorname{Singular}(\mathcal{G})$. Similarly, we also apply the algorithm Merge from Theorem 3.4. Note that only Step 3 involves vertices from $A$.

\section{Procedure Left-B-growth}

Step 1. For $j$ from $t$ down to 1 do the following: $C:=\operatorname{Singular}\left(B \cup C_{j}\right)$, if $C$ is a cycle then $B:=C$ (otherwise, $B$ is not changed). Let $I=\left\{i_{1}, \ldots, i_{k}\right\}$ be the set of subscripts of the cycles in $\mathcal{F}$ which have been merged with a current $B$ in the loop above. If $I=\emptyset$ then set $\mu:=t+1, \mathcal{H}:=\mathcal{F} \cup B$ and go to Step 3. Set $\mu:=\min I$. If $|I|=t$, then return $\mathcal{F}^{\prime}:=B$.

Step 2. While there is $j \in\{\mu+1, \mu+2, \ldots, t\}-I$ do the following: $B:=\operatorname{Merge}\left(B \cup C_{j}\right)$, $I:=I \cup\{j\}$. If $\mu=1$ then return $\mathcal{F}^{\prime}:=B$.

After the execution of Step 2 we have obtained the cycle subgraph $\mathcal{H}=C_{1} \cup C_{2} \cup$ $\ldots \cup C_{\mu-1} \cup B$. (Below we prove that every application of Merge to a subgraph $B \cup C_{j}$ in Step 2 results in a new cycle $B$ ).

Step 3. Apply only the first iteration of Singular to $\mathcal{H}$ in $D^{\prime}$ (recall Note 3.8). If the output of the iteration is $\mathcal{H}$ itself, then return $\mathcal{H}$. Otherwise, Singular has replaced two or more cycles from $\mathcal{H}$ by one new cycle $C$. If $V(B) \cap X \nsubseteq V(C)$, i.e. $B$ was not involved in this merging, then let $B:=\operatorname{Merge}(B \cup C)$ (as we shall prove later, $B$ will, in fact, be a cycle after this step). Otherwise let $B:=C$ Add the subscripts of those cycles $C_{j}(j<\mu)$, merged into $C$ above, to the set $I, \mu:=\min I$ and go to Step 2 .

First note that the call $\operatorname{Singular}\left(B \cup C_{j}\right)$ may release some vertices from $\bar{X}$ which can be used to merge other cycles $C_{p}, C_{q}$ (for some $1 \leq p<q \leq t$ ). This is why we may need to repeat Step 2 and Step 3.

Now we prove the correctness of Left-B-growth. Clearly, the procedure is correct if every call involving Merge in Steps 2 and 3 provides us with a new cycle $B$. Below we show that this is indeed so.

Assume that just after execution of Step $1|I|<t$, i.e. there exists an index $j \in\{1,2, \ldots, t\}-I$. This means that in the $(t-j+1)^{\prime}$ th execution of Step 1 LeftB-growth has tried to merge $C_{j}$ and the cycle $B=B_{j}$, existing at that moment of time, but has not succeeded because $C_{j} \cup B_{j}$ is $X$-singular in $D\left\langle C_{j} \cup B_{j}\right\rangle$ (see Note 3.8). Thus, by Definition 3.5, either $C_{j} \simeq B_{j}$ or $B_{j} \simeq C_{j}$. However, $B_{j} \simeq C_{j}$ is impossible since before the algorithm starts, the vertex $q_{j}$ strongly dominates $V\left(B_{j}\right) \subseteq$ 
$V\left(C_{j+1}\right) \cup \ldots \cup V\left(C_{t}\right) \cup V(B)$. Thus $C_{j} \simeq>B_{j}$. If $C_{j} \Rightarrow B_{j}$, then clearly $V\left(C_{j}\right) \Rightarrow w_{j}$ for all $w_{j} \in V\left(B_{j}\right)$. Otherwise let $b \rightarrow c$ be an arc from $B_{j}$ to $C_{j}$. By Theorem 3.7 and Proposition 3.6, $V\left(C_{j}\right) \Rightarrow b^{+}$and $b^{+} \in X$. Thus we have shown that $B_{j}$ has an $X$-vertex $w_{j}$ such that $V\left(C_{j}\right) \Rightarrow w_{j}$. Observe that the vertex $w_{j}$ will remain in the current $B$ till the end of the execution of the algorithm (see Note 3.8).

Now we prove that every application of Merge to the subgraph $B \cup C_{j}$ in Step 2 results in a new cycle $B$. By Theorem 3.4, it is sufficient to show that one of the alternatives (A)-(C) hold for the cycles $B, C_{j}$. We have proved the existence of a vertex $w_{j} \in X \cap B$ strongly dominated by $C_{j}$. On the other hand, $B$ contains the vertex $q_{\mu}$ which strongly dominates $C_{j}$. Therefore alternative (B) holds and, by Theorem 3.4, Merge will merge $B$ and $C_{j}$ into one cycle.

It remains to prove that every call $B:=\operatorname{Merge}(B \cup C)$ in Step 3 results in a cycle $B$. Since $V(C) \cap V(B)=\emptyset$ and since any subset of cycles of an $X$-singular cycle subgraph is $X$-singular (as always with respect to the corresponding induced subgraphs), $C$ must contain some vertices from $\bar{X}$, i.e. vertices that where left out in a previous merging using Singular (recall that $\mathcal{F}$ is $X$-singular and we use only one iteration of Singular in Step 3). Thus, since $C$ contains a vertex $q_{l}$ (for some $l \leq \mu-1$ ) which strongly dominates $B$ and a vertex $u \in \bar{X}$, we see (by Note 4.3) that alternative (C) of Theorem 3.4 holds for the pair $C, B$ after deleting possible arcs from $u$ to $B$. Hence, by Theorem 3.4, Merge will indeed return a cycle as desired.

It is easy to check that, by Theorem 3.7, the complexity of our procedure is as claimed. Indeed, the performance of Step 1 takes $O\left(n^{4}\right)$ time by Theorem 3.7 and the fact that $t<n$. In Steps 2 and 3, each call of Merge or the first iteration of Singular either results in fewer cycles to be considered or halts the procedure. Again, $t<n$ and Theorems 3.4 and 3.7 imply $O\left(n^{4}\right)$ time for the total performance of Steps 2 and 3 . $\diamond$.

We shall now prove Theorem 4.6, by showing that the following algorithm Leftshift returns the desired cycle subgraph. In the algorithm we use procedure Left-B-growth from the previous lemma and the following procedure Right-B-growth. It is easy to check that the complexity of Right-B-growth is $O\left(n^{5}\right)$.

\section{Procedure Right-B-growth}

Input: A digraph $D$, a cycle $B$ and a cycle subgraph $\mathcal{R}$ of $D-V(B)$.

Output: A cycle $B^{\prime}$ and a cycle subgraph $\mathcal{R}^{\prime}$ such that $X \cap V(B \cup \mathcal{R}) \subseteq V\left(B^{\prime} \cup \mathcal{R}^{\prime}\right)$ and $V(B) \subseteq V\left(B^{\prime}\right)$. Furthermore, there is no 1-path-cycle subgraph $P^{\prime} \cup C_{1} \cup \ldots \cup C_{l}$ of $D-V\left(B^{\prime}\right)$ covering $X \cap V\left(\mathcal{R}^{\prime}\right)$ with the property that $P^{\prime}$ is an $(a, b)$-path and there exists some $q \in V\left(B^{\prime}\right)$ such that $q \rightarrow a$ and $b \rightarrow q^{+}$.

Step 1. Using Lemma 3.2, for every $q \in V(B)$, try to construct a 1-path-cycle subgraph $P \cup C_{1} \cup \ldots \cup C_{s}$ of $D-V(B)$ covering $X \cap V(\mathcal{R})$ such that the path $P$ terminates at a vertex dominating $q^{+}$and starts at a vertex dominated by $q$. If such a subgraph exists, then set $B:=B\left[q^{+}, q\right] P q^{+}$and $\mathcal{R}:=C_{1} \cup \ldots \cup C_{s}$ and go to the beginning of Step 1.

Step 2. Return $B^{\prime}:=B$ and $\mathcal{R}^{\prime}:=\mathcal{R}$. 


\section{Algorithm Leftshift}

Step 1. Let $\mathcal{F}^{\prime}=C_{1}^{\prime} \cup \ldots \cup C_{l}^{\prime}$ be an $X$-singular subgraph of $D$ which covers $X$. If $l=1$ then return $\mathcal{F}:=C_{1}^{\prime}$; halt. Set $\mathcal{A}:=\emptyset, B:=C_{1}^{\prime}, \mathcal{R}=C_{2}^{\prime} \cup \ldots \cup C_{l}^{\prime}, a:=0$ and let $p_{1}$ be any vertex in $V_{\chi_{R}\left(C_{1}^{\prime}\right)} \cap V\left(C_{1}^{\prime}\right)$.

Step 2. Call Right-B-growth with input $D-V(\mathcal{A}), B$ and $\mathcal{R}$. Let the output be $B^{\prime}$ and $\mathcal{R}^{\prime}$. Set $B:=B^{\prime}, \mathcal{R}:=\mathcal{R}^{\prime}$. If $\mathcal{A}=\emptyset$ and $\mathcal{R}=\emptyset$, then return $\mathcal{F}:=B$; halt.

Step 3 If $\bar{X} \neq \emptyset$, the $\operatorname{arc} x_{\alpha} \rightarrow x_{\beta}$ is on $B$ and $\mathcal{R} \neq \emptyset$, then sucessively merge each cycle of $\mathcal{R}$ into $B$, using Merge. Let $B^{*}$ denote the cycle obtained this way (Note that $\left.V\left(B^{*}\right)=V(B) \cup V(\mathcal{R})\right)$. Let $B:=B^{*}$ and $\mathcal{R}:=\emptyset$.

Step 4. If $\mathcal{A}=\emptyset$, then go to Step 6. Otherwise $\left(\mathcal{A}=Z_{1} \cup \ldots \cup Z_{a}\right)$, call Left-B-growth for $\mathcal{A} \cup B$ with $q_{i}=p_{i}, i=1,2, \ldots, a$ (recall that $q_{i}$ is defined in Lemma 6.2) and $A=V(D)-\left(V(\mathcal{A} \cup B \cup \mathcal{R})\right.$, to convert it into an $X$-singular cycle subgraph $\mathcal{F}^{*}$ of $D\langle V(\mathcal{A} \cup B)\rangle$ covering $X \cap V(\mathcal{A} \cup B)$, such that $\mathcal{F}^{*}=Z_{1} \cup \ldots \cup Z_{e} \cup B^{\prime}$ for some $e \in\{0,1, \ldots, a\}$ (see Lemma 6.2). If $\mathcal{R}=\emptyset$, then return $\mathcal{F}:=\mathcal{F}^{*}$; halt.

Step 5. If $e<a$ then set $B:=B^{\prime}, \mathcal{A}:=Z_{1} \cup \ldots \cup Z_{e}, a:=e$ and go to Step 2.

Step 6. Use Singular to obtain an $X$-singular cycle subgraph $\mathcal{H}=H_{1} \cup \ldots \cup H_{h}$ (of $D-V(\mathcal{A} \cup B))$ from $\mathcal{R}$. If $h \geq 2$, then let $p_{a+2}$ be any vertex of $V_{\chi_{R}\left(H_{1}\right)} \cap V\left(H_{1}\right)$. (Here $\chi_{R}\left(H_{1}\right)$ is defined with respect to $\mathcal{H}$.)

Step 7. Let $Z_{a+1}:=B, \mathcal{A}:=Z_{1} \cup \ldots \cup Z_{a+1}, B:=H_{1}, \mathcal{R}:=H_{2} \cup \ldots \cup H_{h}$ and let $a:=a+1$. If $\mathcal{R}=\emptyset$, then go to Step 4 , otherwise go to Step 2 .

Below we prove the correctness of Leftshift and determine its time complexity.

To prove the correctness of the algorithm it is enough to prove that, in every performance of Step $4, p_{i}$ is a proper choice for $q_{i}$ in Lemma 6.2 for all $i=1, \ldots, a$ (that is we can actually apply the algorithm Left- $B$-growth as we claim), and that the output of the algorithm, $\mathcal{F}=C_{1} \cup \ldots \cup C_{t}$, is $X$-leftshifted. The first part follows from the following claim.

Claim: The following properties hold during the execution of Leftshift:

(i) If $V(B) \cap \bar{X}$ becomes non-empty (in Step 2 or Step 4) this will lead to the execution of Step 3 and hence render $\mathcal{R}=\emptyset$.

(ii) At all times during the execution of Leftshift we have $V(\mathcal{A}) \subseteq X$.

(iii) The vertex $p_{i}$ is a proper choice for $q_{i}$ in Lemma 6.2 for all $i=1, \ldots, a$

Proof of the claim: Suppose $V(B) \cap \bar{X}$ becomes non-empty during the algorithm. If this happens (for the first time) in Step 4, then Step 2 will be executed just after this (via Step 5). Otherwise $V(B) \cap \bar{X}$ becomes non-empty in Step 2. Now consider that execution of Step 2 when $V(B) \cap \bar{X}$ becomes non-empty for the first time, or 
has just become non-empty in Step 4. Note that at this point we have $V(\mathcal{A}) \subseteq X$, since vertices are only added to $\mathcal{A}$ in Step 7 and after Step $5 \mathcal{A} \subset X$, by Lemma 4.4. Furthermore, we claim that the arc $x_{\alpha} \rightarrow x_{\beta}$ will be an arc of $B$ before the current execution of Step 2 finishes. Indeed, By Lemma 4.5, $x_{\alpha} \rightarrow x_{\beta}$ was an arc of $C_{l}$ after Step 1 and hence is currently on some cycle $C^{\prime}$ in $\mathcal{R} ; B$ has a vertex of $\bar{X}$, thus, the proof of Lemma 6.1 shows that Right-B-growth will merge $B$ and $C^{\prime}$. Lemma 6.1 implies that when $x_{\alpha} \rightarrow x_{\beta}$ has become an arc of $B$, then Step 2 will either terminate with $\mathcal{R}=\emptyset$, or with $V(\mathcal{R}) \subseteq X$. In the last case Step 3 will be executed and since $B$ contains a vertex $p$ that strongly dominates every vertex in $\mathcal{R}$ (one of the $p_{i}$ 's defined in an earlier execution of Step 7; compare with proposition 3.6) and $B$ also contains the vertex $x_{\alpha}$ which is strongly dominated by every vertex in $\mathcal{R}$, it follows from Theorem 3.4 (alternative $(\mathrm{C})$ holds for $B$ and every cycle of $\mathcal{R}$ ) that after excution of Step 3 we have $\mathcal{R}=\emptyset$. Observe that (i) and (ii) follow easily from this, since after $\mathcal{R}$ becomes empty $\mathcal{A}$ will not be redefined anymore.

To prove (iii), we first observe that at all executions of Step 4, except the last one we have $V(\mathcal{A} \cup B) \subseteq X$. This implies that no vertex was lost when any earlier execution of Step 4 was performed. Secondly, no $p_{i}$ is defined after the (unique) execution of Step 3. Thus it follows from the choice of $p_{a+2}$ in Step 7 and the definition of $p_{1}$ that (iii) holds (note that when some cycles of the current $\mathcal{A}$ are merged into $B$ in Step 4 only the vertex $p_{i}$ with the smallest index among these will survive as a $p_{i}$ that can later be referred to). This completes the proof of the claim.

We now prove that the output of the algorithm, $\mathcal{F}=C_{1} \cup \ldots \cup C_{t}$, is $X$-leftshifted. Suppose that $\mathcal{F}$ is not $X$-leftshifted. Then there exist $1 \leq i<j \leq t$ and a 1-path-cycle subgraph $\mathcal{F}^{\prime}=P \cup M_{1} \ldots \cup M_{k}$ such that $X \cap V\left(C_{j}\right) \subseteq V(\mathcal{F}) \cap V\left(\mathcal{F}^{\prime}\right) \subseteq V\left(C_{j}\right)$, $P$ is a $(u, v)$-path and there exists $w \in V\left(C_{i}\right)$ such that $w \rightarrow u$ and $v \rightarrow w^{+}$.

Consider that performance of Step 7, where $C_{i}$ enters $\mathcal{A}$. Just before this step of the algorithm $B=C_{i}$. Consider also the last performance of Step 2 before the above-mentioned performance of Step 7. Clearly, $B^{\prime}=B=C_{i}$ is the output of RightB-growth in this performance of Step 2. Let $k<i$. By Lemma 4.4 and the fact that every subset of the cycles of a singular cycle subgraph is singular, $V\left(C_{k}\right) \subseteq X$. Thus, by Note 3.8 and part (ii) of the claim above, the cycle $C_{k}$ has not been changed since it entered $\mathcal{A}$. Moreover, $C_{k}$ was constructed before $C_{i}$. Hence, after the above-mentioned performance of Step $2 \mathcal{A}=C_{1} \cup \ldots \cup C_{i-1}$. Thus, the vertices of $\mathcal{F}^{\prime}$ were available to "enlarge" $B$ in the above-mentioned performance of Right-B-growth, contradicting the fact that Right- $B$-growth did not change $B$.

It is easy to see that the complexity of the algorithm is $O\left(\left(n_{2}+1\right) n^{5}\right)$, where $n_{2}$ is the number of executions of Step 2. To bound $n_{2}$, we introduce a potential function $L=a(\mathcal{A})+2|X \cap V(\mathcal{R})|$, where $a(\mathcal{A})$ denotes the number of cycles in $\mathcal{A}$. We can return to Step 2 from either Step 5 or Step 7. If we return from Step 5, then $a(\mathcal{A})$ decreases and $2|X \cap V(\mathcal{R})|$ remains unchanged. If we return from Step 7, then $a(\mathcal{A})$ increases by one, but $2|X \cap V(\mathcal{R})|$ decreases by at least two. Thus, every time we return to Step 2 , the function $L$ decreases. Moreover, $0 \leq L \leq 3 n$. Hence, $n_{2}=O(n)$. Thus, the complexity of the algorithm is $O\left(n^{6}\right)$. 


\section{Open problems and conjectures}

The existence of a polynomial algorithm for the Hamiltonian cycle problem in semicomplete multipartite digraphs indicates the possible existence of a "nice" mathematical characterization of Hamiltonian semicomplete multipartite digraphs. So far we have not managed to find one.

We believe that some more general problems for semicomplete multipartite digraphs $D$ are also polynomial time solvable:

Conjecture 7.1 There are polynomial algorithms for the following problems:

1) Given set $X \subseteq V(D)$ find a cycle covering $X$.

2) Construct a longest cycle in $D$.

3) Given set $X \subseteq V(D)$ find a cycle containing the maximum number of vertices from $X$.

Conjecture 7.1.1 seems quite difficult, even in the case of semicomplete bipartite digraphs it is still open (see [5]). However, the case of extended semicomplete digraphs is solved in [3]. Conjecture 7.1.2 is settled for both semicomplete bipartite digraphs and extended semicomplete digraphs (cf. [16]).

Another interesting problem is to characterize weakly Hamiltonian-connected semicomplete multipartite digraphs, i.e. semicomplete multipartite digraphs having a Hamiltonian path between every pair of vertices. The problem was settled for bipartite semicomplete and extended semicomplete digraphs in [6] and [2], respectively.

\section{References}

[1] R. K. Ahuja, T. L. Magnanti and J. B. Orlin, Network Flows, Prentice Hall, Englewood Clifs, New Jersey (1993).

[2] J. Bang-Jensen, G. Gutin and J. Huang, Weakly Hamiltonian-connected ordinary multipartite tournaments, Discrete Math. 138 (1995) 63-74.

[3] J. Bang-Jensen, G. Gutin and J. Huang, A sufficient condition for a semicomplete multipartite digraph to be Hamiltonian, Discrete Math. 161 (1996) 1-12.

[4] J. Bang-Jensen, G. Gutin and A. Yeo, Hamiltonian cycles avoiding prescribed arcs in tournaments, Combinatorics, Probability and Computing 11 (1997) 1-7.

[5] J. Bang-Jensen and Y. Manoussakis, Cycles through $k$ vertices and $k$ arcs in bipartite tournaments. Combinatorica 14 (1994) 1-4.

[6] J. Bang-Jensen and Y. Manoussakis, Weakly Hamiltonian-connected vertices in bipartite tournaments, J. Combin. Theory, B 63 (1995) 261-280.

[7] J. Bang-Jensen and C. Thomassen, A polynomial algorithm for the 2-path problem for semicomplete digraphs. SIAM J. Disc. Math. 5 (1992) 366-376. 
[8] J.A. Bondy and U.S.R. Murty, Graph Theory with Applications, MacMillan Press, 1976.

[9] P. Camion, Chemins et circuits hamiltoniens des graphes complets. C.R. Acad. Sci. Paris 249 (1959) 2151-2152.

[10] J. F. Fink and L. Lesniak-Forster, Graphs for which every unilateral orientation is traceable, Ars Combinatoria 9 (1980) 113-118.

[11] L. R. Ford and D. R. Fulkerson, Flows in Networks, Princeton University Press, Princeton New Jersey (1962).

[12] G. Gutin, A characterization of complete $n$-partite digraphs that have a Hamiltonian path. Kibernetica No. 1 (1988) 107-108 (In Russian).

[13] G. Gutin, A criterion for complete bipartite digraphs to be Hamiltonian. Vestsi Acad. Navuk BSSR Ser. Fiz.-Mat. Navuk No. 1 (1984) 99-100 (In Russian).

[14] G. Gutin, Characterizations of vertex pancyclic and pancyclic ordinary complete multipartite digraphs Discrete Math. 141 (1995) 153-162.

[15] G. Gutin, Cycles and paths in semicomplete multipartite digraphs, theorems and algorithms: a survey. J. Graph Theory 19 (1995) 481-505.

[16] G. Gutin, Paths and cycles in digraphs. Ph.D thesis, Tel Aviv Univ., 1993.

[17] R. Häggkvist and Y. Manoussakis, Cycles and paths in bipartite tournaments with spanning configurations. Combinatorica 9 (1989) 33-38.

[18] Y. Manoussakis, A linear time algorithm for finding Hamiltonian cycle in tournaments. Discrete Appl. Math. 36 (1992) 199-202.

[19] Y. Manoussakis and Z. Tuza, Polynomial algorithms for finding cycles and paths in bipartite tournaments. SIAM J. Disc. Math. 3 (1990) 537-543.

[20] A. Yeo, Cycles in semicomplete multipartite digraphs, Midterm report for Ph. D. study at Department of Mathematics and Computer Science at Odense University, available at http://www.imada.ou.dk/ gyeo/Projects/project.html

[21] A. Yeo, One-diregular subgraphs in semicomplete multipartite digraphs, Journal of Graph Theory 24 (1997) 1-11.

[22] C. Q. Zhang, Hamiltonian paths in multipartite oriented graphs, Annals of Discrete Mathematics 41 (1989) 499-514. 